\title{
New baryonic and mesonic observables from NA61/SHINE
}

\author{
Antoni Marcinek ${ }^{1,2, a}$ for the NA49 and NA61/SHINE collaborations \\ ${ }^{1} \mathrm{H}$. Niewodniczański Institute of Nuclear Physics, Polish Academy of Sciences, PL-31342 Kraków, Poland \\ 2Jagiellonian University, Kraków, Poland
}

\begin{abstract}
One of the main objectives of the NA61/SHINE experiment at the CERN SPS is to study properties of strongly interacting matter. This paper presents new results on observables relevant for this part of the NA61/SHINE programme. These include the first ever measurements of $\phi$ meson production in $\mathrm{p}+\mathrm{p}$ collisions at 40 and $80 \mathrm{GeV}$, and most detailed ever experimental data at $158 \mathrm{GeV}$. This contribution demonstrates the superior accuracy of the present dataset with respect to existing measurements. The comparison of $\mathrm{p}+\mathrm{p}$ to $\mathrm{Pb}+\mathrm{Pb}$ collisions shows a non-trivial system size dependence of the longitudinal evolution of hidden strangeness production, contrasting with that of other mesons. Furthermore, proton density fluctuations are investigated as a possible order parameter of the second order phase transition in the neighbourhood of the critical point $(\mathrm{CP})$ of strongly interacting matter. An intermittency analysis is performed of the proton second scaled factorial moments in transverse momentum space. A previous analysis of this sort revealed significant power-law fluctuations for the "Si" $+\mathrm{Si}$ system at $158 \mathrm{~A} \mathrm{GeV}$ measured by the NA49 experiment. The fitted power-law exponent was consistent within errors with the theoretically expected critical value, a result suggesting a baryochemical potential in the vicinity of the $\mathrm{CP}$ of about $250 \mathrm{MeV}$ [24]. The analysis will now be extended to NA61/SHINE systems of similar size, $\mathrm{Be}+\mathrm{Be}$ and $\mathrm{Ar}+\mathrm{Sc}$, at $150 \mathrm{~A} \mathrm{GeV}$. Finally, spectator-induced electromagnetic (EM) effects on charged meson production are being studied and bring information on the space-time position of the pion formation zone, which appears to be much closer to the spectator system for faster pions than for slower ones. On that basis, we demonstrate that the longitudinal evolution of the system at CERN SPS energies may be interpreted as a pure consequence of local energymomentum conservation.
\end{abstract}

\section{Introduction}

NA61/SHINE [1] is a fixed target experiment at the CERN SPS accelerator complex. Its strong interactions programme is a continuation and extension of the NA49 experiment [2], following NA49's discovery of the onset of deconfinement in $\mathrm{Pb}+\mathrm{Pb}$ collisions at $30 \mathrm{~A} \mathrm{GeV}$ beam momentum [3, 4]. NA61/SHINE performs the first two-dimensional scan of the phase diagram of strongly interacting matter (SIM) by varying the momentum and size of the colliding nuclei, in search for the critical point of SIM and to study properties of the onset of deconfinement. Of interest to this programme are thus strangeness production (section 2), fluctuations of baryon density (section 3 ) and space-time properties of the medium created in heavy ion collisions (section 4).

ae-mail: antoni.marcinek@gmail.com 


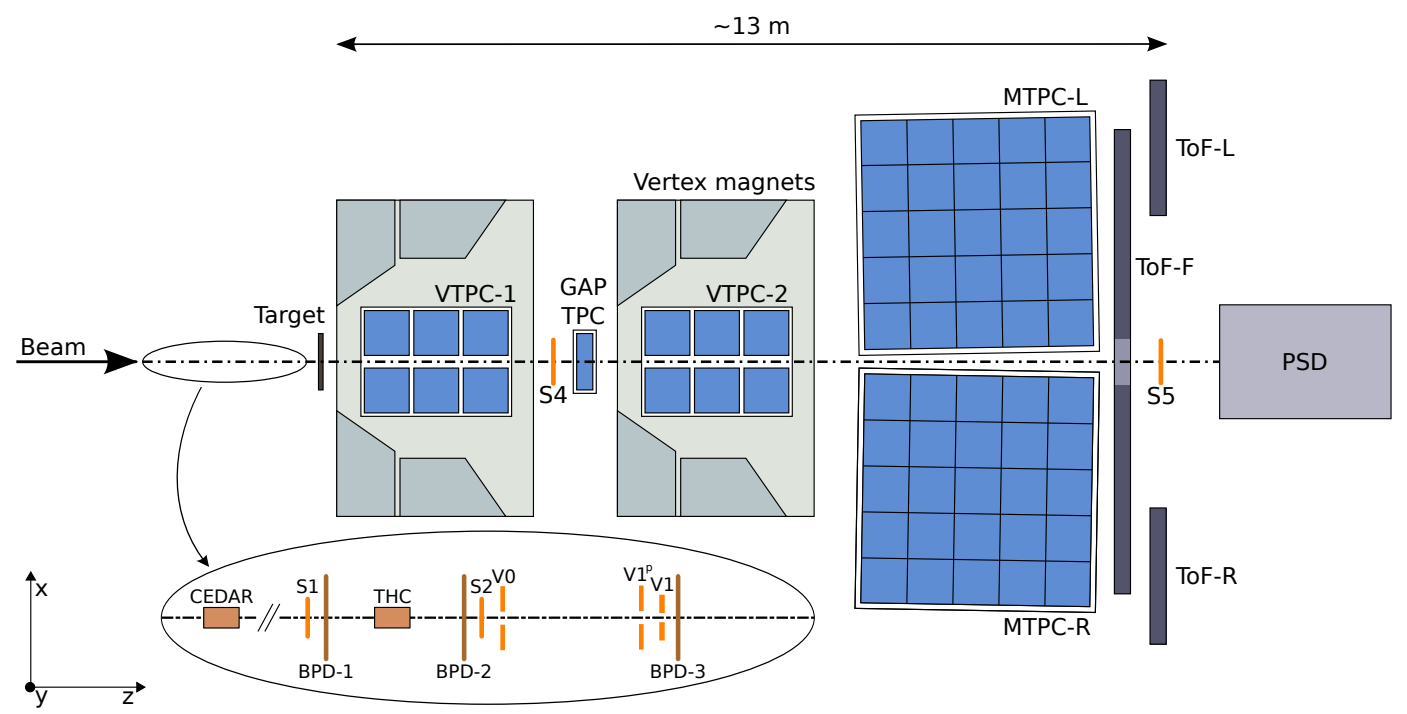

Figure 1. Schematic layout of the NA61/SHINE detector system [1] (horizontal cut in the beam plane, not to scale). Also outlined are the coordinate system used in the experiment and the configuration of beam detectors used with secondary proton beams for $\mathrm{p}+\mathrm{p}$ interactions for which $\phi$ meson production results are presented.

The main parts of the NA61/SHINE detector system (Fig. 1) were inherited from the NA49 experiment. Large volume Time Projection Chambers (TPC), two of them immersed in vertical magnetic fields, allow precise measurement of momenta of charged particles down to $p_{T}=0$ and provide particle identification (PID) via the $\mathrm{dE} / \mathrm{dx}$ method. Time of Flight (ToF) walls complement PID capabilities. The Projectile Spectator Detector (PSD), a hadron calorimeter, measures forward-going energy allowing to estimate the centrality of nuclear collisions.

\section{Hidden strangeness production in proton-proton collisions}

The $\phi$ meson, consisting of $s$ and $\bar{s}$ valence quarks, is a particle with hidden strangeness. Motivation for measurement of its production in $\mathrm{p}+\mathrm{p}$ collisions is twofold. First, it is useful in itself to constrain hadron production models. Second, it may serve as reference for $\mathrm{Pb}+\mathrm{Pb}$ measurements at the same energies to identify strangeness-related phenomena specific to heavy ion collisions.

In this contribution, differential multiplicities of $\phi$ mesons in $\mathrm{p}+\mathrm{p}$ interactions at 40,80 and $158 \mathrm{GeV}$ beam momenta, as a function of rapidity y and transverse momentum $p_{T}$, are obtained from invariant mass spectra in the $\phi \rightarrow K^{+} K^{-}$decay channel. In Fig. 2 one can see $p_{T}$ spectra in rapidity bins for the $158 \mathrm{GeV}$ data set. Similar double-differential spectra are also measured for $80 \mathrm{GeV}$, while due to low track statistics, only single-differential analysis, separately in y and in $p_{T}$, was feasible for the $40 \mathrm{GeV}$ data. These are the first ever differential measurements of $\phi$ production at 40 and $80 \mathrm{GeV}$ and the first double-differential measurements at $158 \mathrm{GeV}$.

Transverse momentum spectra are compared to three models: Epos 1.99 [5, 6] from the CRMc 1.6.0 package [7], Pyтhia 6.4.28 [8] and URQMD 3.4 [9, 10]. Model predictions in Fig. 2 are normalized to the integral of the data in each rapidity bin to focus only on shape comparison. One sees that PүтнIA describes well the shape, while the spectra from URQMD are too hard and those from Epos too soft. 


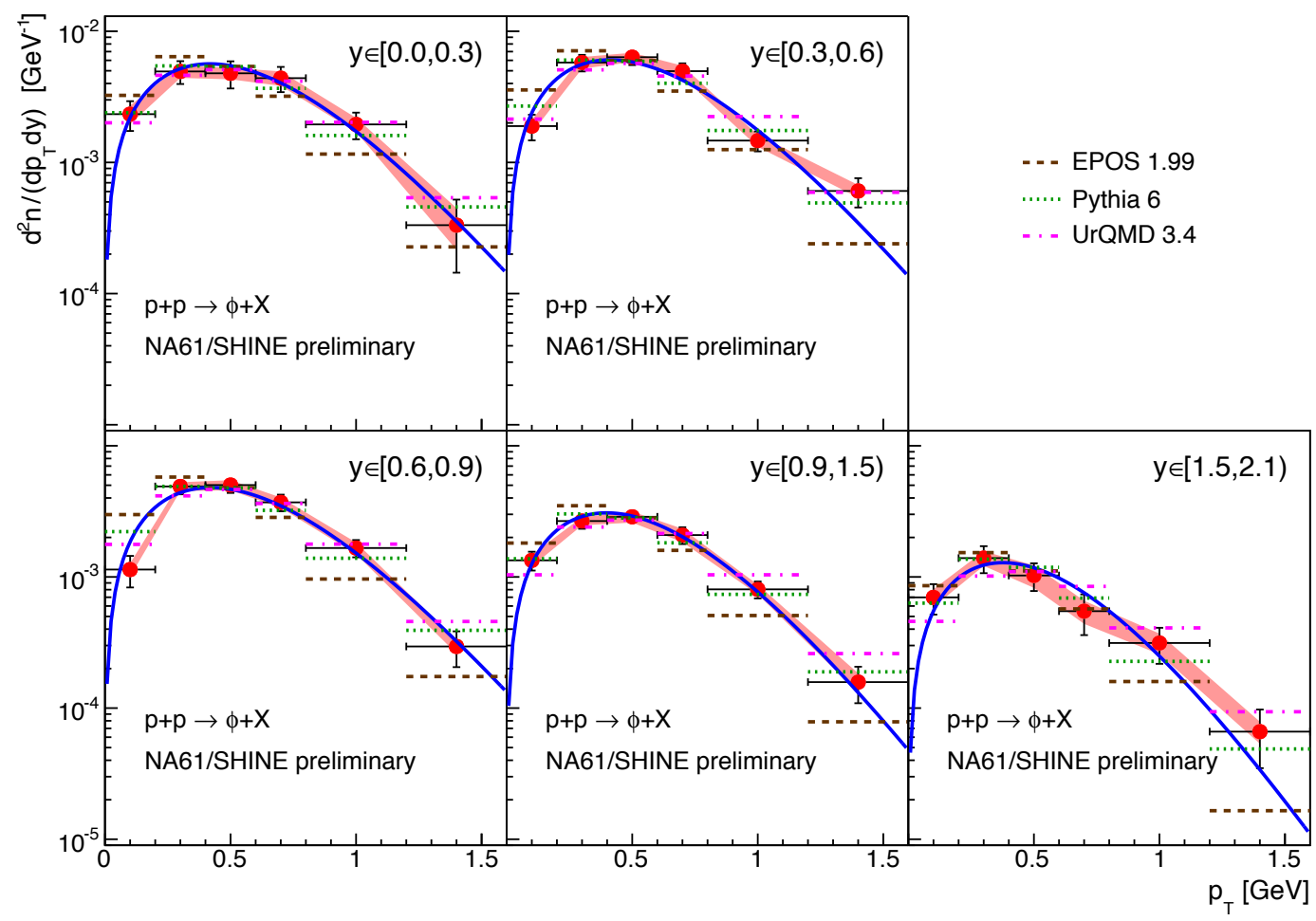

Figure 2. Transverse momentum spectra in rapidity bins of $\phi$ mesons in $\mathrm{p}+\mathrm{p}$ collisions at $158 \mathrm{GeV}$ beam momentum $\left(\sqrt{s_{N N}}=17.3 \mathrm{GeV}\right.$ ) with statistical (vertical lines) and systematic (bands) uncertainties. Horizontal lines give $p_{T}$ bin sizes. Regarding models and the fitted function see text.

Having the double differential spectra it is possible to calculate the single differential spectra of rapidity integrated over $p_{T}$ by summation of the measured $p_{T}$ region and extrapolation to large $p_{T}$. The latter is done using a thermally motivated fit function $p_{T} e^{m_{T} / T}$ (thick curves in Fig. 2). The unmeasured tail contribution is smaller than $1 \%$ for all y bins in Fig. 2.

The resulting rapidity distributions are shown in Fig. 3 for all studied energies. Again the shape comparison with the three models is performed using the same normalization scheme as for $p_{T}$ spectra. In this case both Epos and URQMD predict shapes comparable to that of the data, while PYTHIA produces a distribution which is too narrow. For the largest energy, single differential measurements in narrower rapidity range, are available from NA49 [11]. It is clear that the two measurements are consistent.

In order to calculate total $\phi$ multiplicities, distributions are fitted with Gaussian functions ${ }^{1}$ (solid curves in Fig. 3). Again summation of the measured spectrum is done and the unmeasured tail contribution from the fit is added (3\% to 7\% depending on energy). Then the result is doubled to account for the backward hemisphere from collision symmetry. The energy dependence of the obtained total $\phi$ yields is shown in Fig. 4 and compared to world data [11-15]. Clearly NA61/SHINE results are

\footnotetext{
${ }^{1}$ It should be noted that for $\phi$ meson rapidity distributions a double Gaussian (dashed curves in Fig. 3) does not provide any advantage over a single Gaussian function contrary to the case of charged hadrons in $\mathrm{p}+\mathrm{p}$ [18].
} 

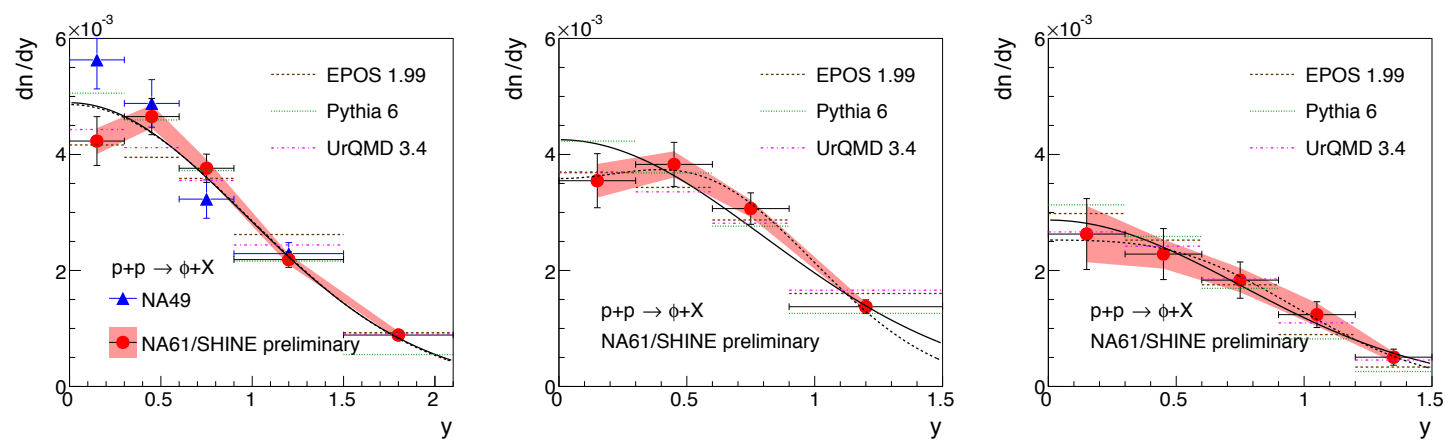

Figure 3. Rapidity distributions of $\phi$ mesons in $\mathrm{p}+\mathrm{p}$ collisions at beam momenta of $158 \mathrm{GeV}$ (left, $\sqrt{s_{N N}}=$ $17.3 \mathrm{GeV}$ ), $80 \mathrm{GeV}$ (middle, $\sqrt{s_{N N}}=12.3 \mathrm{GeV}$ ) and $40 \mathrm{GeV}\left(\right.$ right, $\sqrt{s_{N N}}=8.8 \mathrm{GeV}$ ) with statistical (vertical lines) and systematic (bands) uncertainties. Horizontal lines give y bin sizes. NA49 data points come from Ref. [11]. Regarding models and the fitted function see text.

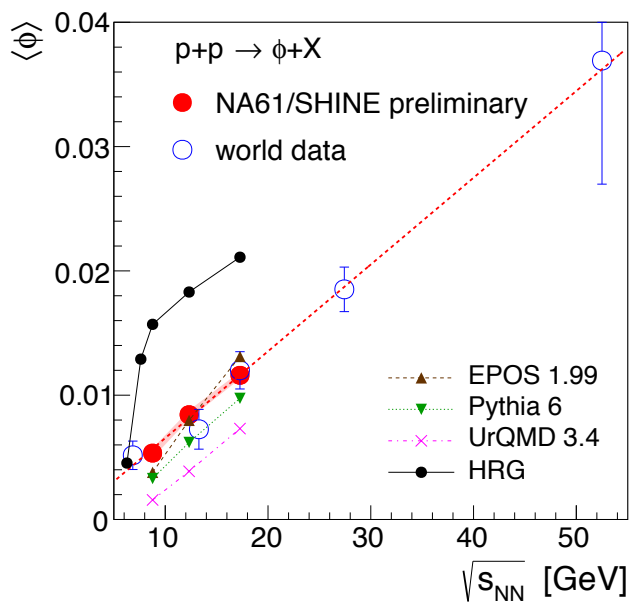

Figure 4. Energy dependence of total yields of $\phi$ mesons in $\mathrm{p}+\mathrm{p}$ collisions. World data come from Refs. [11-15]. Total uncertainties are shown; for preliminary NA61/SHINE results total uncertainties are smaller than markers. Red dashed line is to guide the eye. Regarding models see text.

consistent with the world data, but are much more accurate. The figure also shows a comparison of $\phi$ yields with models, including the hadron resonance gas (HRG [16]) statistical model. It is apparent that while Epos describes the data reasonably well (although the rise with collision energy is too fast), all other models fail with URQMD underestimating and HRG overestimating the yield by about a factor of 2 .

Figure 5 demonstrates the enhancement of strange meson production in $\mathrm{Pb}+\mathrm{Pb}$ collisions compared to $\mathrm{p}+\mathrm{p}$ reactions. Data on $\phi$ in $\mathrm{Pb}+\mathrm{Pb}$ and other mesons in both systems come from Refs. [3, 4, 17, 18]. Multiplicities of strange mesons are divided by those of pions to take out the "trivial" effect of the size of the colliding system. In case of the $\phi$, the mean total yield of pions is used which was calculated as Ref. [17]:

$$
\langle\pi\rangle=\frac{3}{2}\left(\left\langle\pi^{+}\right\rangle+\left\langle\pi^{-}\right\rangle\right)
$$



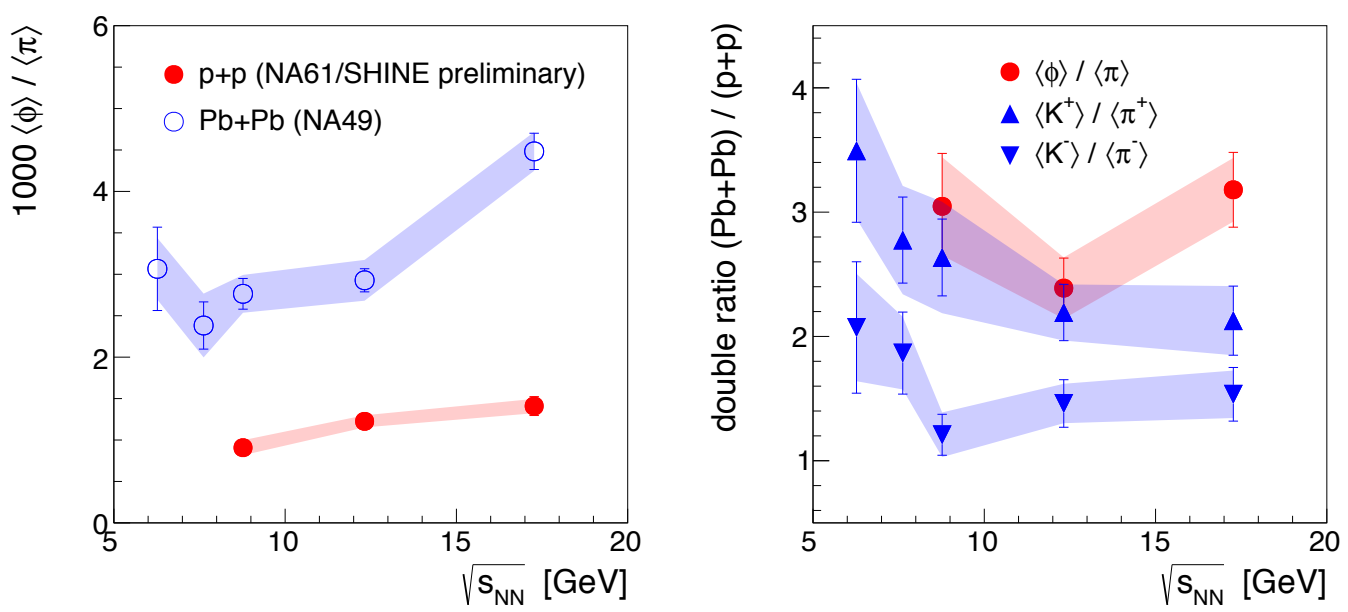

Figure 5. Energy dependence of (left) ratios of total yields of $\phi$ mesons to mean total yields for pions Eq. (1) in $\mathrm{p}+\mathrm{p}$ and $\mathrm{Pb}+\mathrm{Pb}$, (right) double ratios (see text), with statistical (vertical lines) and systematic (bands) uncertainties. Full circles correspond to results of this analysis, $\mathrm{Pb}+\mathrm{Pb}$ data come from NA49 [3, 4, 17], while $\mathrm{p}+\mathrm{p}$ kaon and pion data are taken from Ref. [18].

It is evident from Fig. 5 (left), that the $\phi / \pi$ ratio increases with energy for both systems. The enhancement from $\mathrm{p}+\mathrm{p}$ to central $\mathrm{Pb}+\mathrm{Pb}$ collisions is about a factor 3 .

Dividing the $\mathrm{Pb}+\mathrm{Pb}$ by the $\mathrm{p}+\mathrm{p}$ ratios one gets the double ratio:

$$
\text { double ratio }(\langle\phi\rangle /\langle\pi\rangle)=\frac{(\langle\phi\rangle /\langle\pi\rangle)_{\mathrm{Pb}+\mathrm{Pb}}}{(\langle\phi\rangle /\langle\pi\rangle)_{\mathrm{p}+\mathrm{p}}},
$$

which is shown by the full circles in Fig. 5 (right). The analogous ratio for kaons is also plotted in Fig. 5 (right). It is apparent that the enhancement of $\phi$ production is comparable to that of $K^{+}$, while the enhancement for $K^{-}$is systematically smaller. Furthermore the enhancement of $\phi$ production is about the square of the $K^{-}$enhancement, consistent with the hypothesis of strangeness enhancement in a parton phase of the collision.

Finally, Fig. 6 shows the widths $\sigma_{\mathrm{y}}$ of rapidity distributions of $\phi$ mesons and various other particles in $\mathrm{p}+\mathrm{p}$ and central $\mathrm{Pb}+\mathrm{Pb}$ collisions $[3,4,11,17-20]$ as a function of beam rapidity $\mathrm{y}_{\text {beam }}$ in the centreof-mass frame. Widths are derived from single or double Gaussian fits depending on the particle species. It is striking that all particles in both colliding systems, except $\phi$ in $P b+P b$ follow the same trend: $\sigma_{\mathrm{y}}$ is proportional to ybeam in the studied range, with the same slope of increase with energy. While the effect was already known from Ref. [17], the new NA61/SHINE results emphasize the peculiarity of the system size dependence of the longitudinal evolution of $\phi$ production, contrasting with that of other mesons.

It should be noted, that the behaviour of $\sigma_{\mathrm{y}}$ for $\phi$ mesons in $\mathrm{Pb}+\mathrm{Pb}$ collisions is qualitatively consistent with rescattering of kaons from $\phi$ mesons decaying inside the fireball $[17,21]$. Such kaons no longer contribute to the signal peak in the invariant mass spectrum. It is more likely to take place for slow kaons (i.e. those coming from low rapidity $\phi$ mesons), which travel longer through the fireball. Therefore, the $\phi$ rapidity spectrum gets depleted and this loss is the highest at midrapidity and decreases with increasing y values. So the spectrum becomes wider due to the rescattering. The 


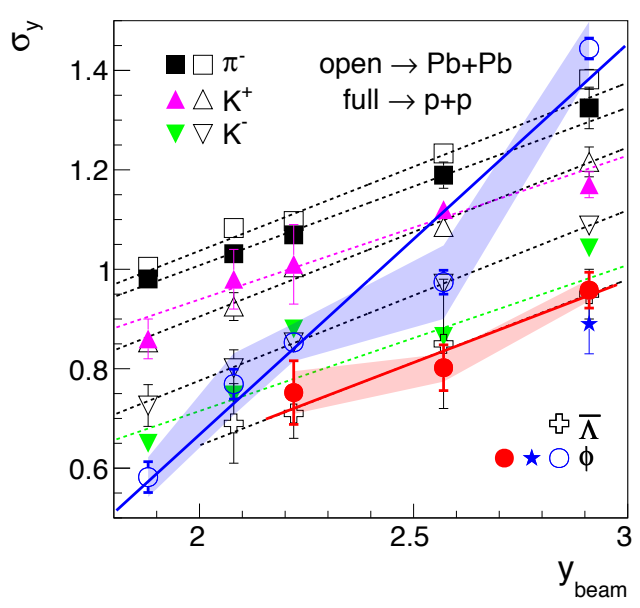

Figure 6. Widths of rapidity distributions of various particles in $\mathrm{p}+\mathrm{p}$ (full symbols) and central $\mathrm{Pb}+\mathrm{Pb}$ (open symbols) collisions as a function of beam rapidity, with statistical (vertical lines) and systematic (bands) uncertainties. Full circles are results of this analysis, the star is a NA49 measurement in $\mathrm{p}+\mathrm{p}$ [11], other $\mathrm{p}+\mathrm{p}$ points come from NA61/SHINE $[18,19] . \mathrm{Pb}+\mathrm{Pb}$ data are from NA49 [3, 4, 17, 20]. Lines are fitted to points to guide the eye.

effect should increase with the collision energy, because the higher the energy, the larger and denser the fireball. Moreover, it is natural to assume that the effect would be much stronger in a quark gluon plasma, than in a purely hadronic medium. This shows the importance of investigating the system size dependence of the effect for systems between $\mathrm{p}+\mathrm{p}$ and $\mathrm{Pb}+\mathrm{Pb}$, for which data are collected by NA61/SHINE.

\section{Search for the QCD critical point via intermittency analysis}

In the vicinity of the critical point of strongly interacting matter increased fluctuations in the system are expected in analogy to critical opalescence in liquid-vapour phase transitions. Therefore the procedure of the search for the CP is to scan the phase diagram of SIM by varying energy and size of colliding nuclei and look for a maximum in fluctuation observables.

Among various fluctuation measures, this contribution focuses on local, power-law fluctuations of baryon density. These are studied by calculating second scaled factorial moments $F_{2}$ in transverse momentum space ( $p_{y}$ vs. $p_{x}$ ) of protons in midrapidity:

$$
F_{2}(M) \equiv \frac{\left\langle\sum_{m} n_{m}\left(n_{m}-1\right)\right\rangle}{\left\langle\sum_{m} n_{m}^{2}\right\rangle},
$$

where the $\left(p_{x}, p_{y}\right)$ space is divided into $M$ bins, $n_{m}$ is the number of protons in bin number $m$ and $\langle\ldots\rangle$ denotes averaging over events. For a critical system, $F_{2}$ is expected to scale according to a specific power law with the bin size or equivalently number of bins $M$. This scaling is called intermittency and the corresponding exponent is called the intermittency index $\phi_{2}$ [22]. However, it is also expected that in addition to protons stemming from a critical system, there is some non-critical background. The latter is estimated using event mixing. So finally the correlator

$$
\Delta F_{2}(M)=F_{2}^{\text {data }}(M)-F_{2}^{\text {mix }}(M)
$$

should scale for $M \gg 1$ proportionally to $\left(M^{2}\right)^{\varphi_{2}}$ with intermittency index $\varphi_{2}=5 / 6$ [23]. 

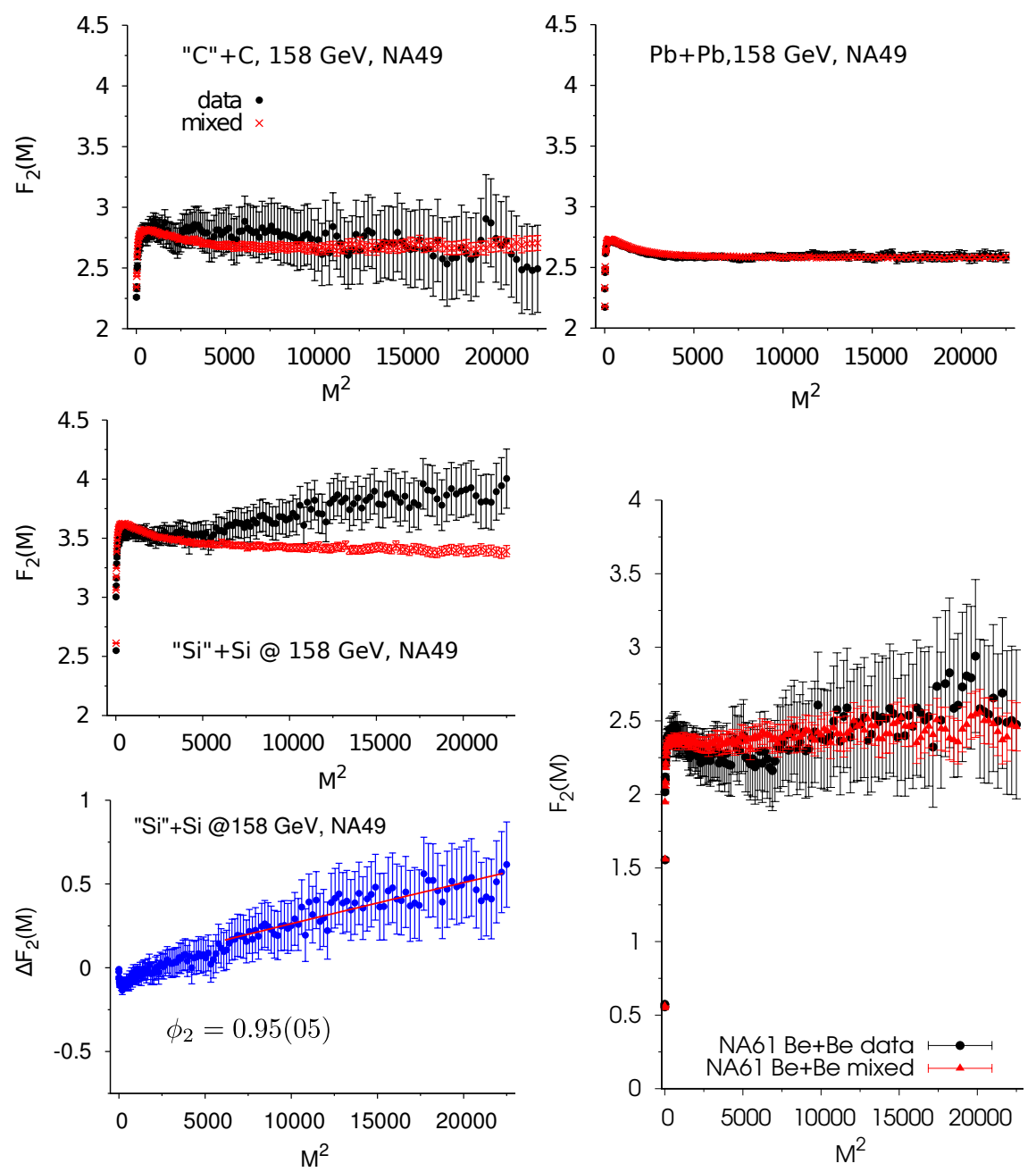

Figure 7. Second scaled factorial moments in transverse momentum space for protons in midrapidity for various colliding systems at top CERN SPS energy, measured by NA49 [24] and NA61/SHINE.

Figure 7 shows $F_{2}(M)$ as measured by NA49 for several systems at $158 A \mathrm{GeV}$ [24]. It is seen that data and mixed events plots overlap for the " $\mathrm{C}$ " $+\mathrm{C}$ and $\mathrm{Pb}+\mathrm{Pb}$ systems ${ }^{2}$, so no intermittency is observed there. In the "Si" $+\mathrm{Si}$ system, on the other hand, there is a clear separation of data and mixed events, so $\Delta F_{2}(M)$ can be calculated and fitted. The intermittency index $\varphi_{2}=0.96_{-0.25}^{+0.38}$ (stat.) \pm 0.16 (syst.) thus obtained is consistent, within uncertainties, ${ }^{3}$ with the theoretical predictions for a critical system. Figure 7 also shows $F_{2}(M)$ for the Be+Be system measured by NA61/SHINE at the top SPS energy. No evidence for intermittency is observed there, consistent with NA49 results, as the $\mathrm{Be}+\mathrm{Be}$ system is lighter than "C" $+\mathrm{C}$ and therefore yet further away from the "Si" $+\mathrm{Si}$ system. Finally, the analysis for the heavier Ar+Sc system in NA61/SHINE is ongoing [25].

\footnotetext{
${ }^{2}$ Here "C" ("Si") actually are a mixture of nuclei with $\mathrm{Z}=6,7(13,14,15)$.

${ }^{3}$ Statistical uncertainty was estimated from the bootstrap method [24].
} 

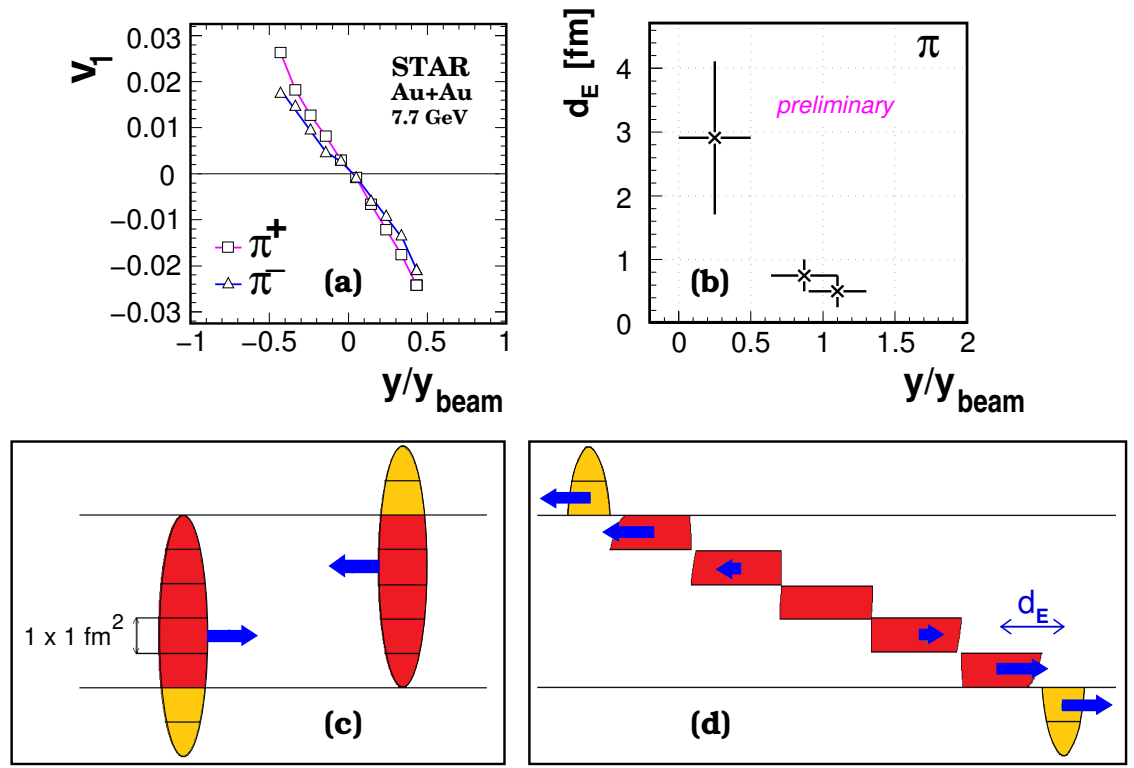

Figure 8. (a) Directed flow for $\pi^{+}$and $\pi^{-}$in Au+Au collisions [27]; original data from Ref. [26]. (b) Dependence of $d_{E}$ on pion rapidity for $\mathrm{Au}+\mathrm{Au}$ and $\mathrm{Pb}+\mathrm{Pb}$ collisions at RHIC BES/CERN SPS energies [30]. (c) "Bricks" of matter considered in the fire streak model of Ref. [31] before the collision. (d) Fire streaks formed after the collision, also redrawn from Ref. [31]. Thick arrows indicate velocity vectors.

\section{Electromagnetic effects in pion emission}

Charged spectators in non-central heavy ion collisions generate electromagnetic fields, which modify trajectories of final state charged particles (most of them are pions). Oppositely charged particles are affected oppositely, leading to charge asymmetries in distributions of produced particles. One example of such asymmetries is charge splitting of directed flow $v_{1}$ visible in Fig. 8(a) for Au+Au collisions measured by the STAR experiment [26]. It was explained by the spectator-induced electromagnetic (EM) effects assuming that the distance $d_{E}$ between the pion formation zone and the spectator was of the order of $3 \mathrm{fm} \mathrm{[27].}$

Taking together results of three similar analyses of EM effects in three different data sets on $\mathrm{Au}+\mathrm{Au}$ and $\mathrm{Pb}+\mathrm{Pb}$ collisions [26, 28, 29], the dependence of $d_{E}$ on pion rapidity, shown in Fig. 8(b), was obtained [30]. It is visible, that faster pions are produced closer to the spectator system.

The latter observation was explained in Ref. [31] in a new, independent realization of the fire streak model [32], sketched in Figs. 8(c) and (d). In the collision centre-of-mass frame, the two incoming nuclei are represented as two continuous, Lorentz-contracted 3D mass distributions defined by the known nuclear density profiles [33]. They are then divided into "bricks" in the transverse plane of the collision. Each brick collides independently with the one from the opposite nucleus, forming a "fire streak". For each pair, local energy-momentum conservation is assumed, which yields the excitation energy $E_{\mathrm{s}}^{*}$ and rapidity $\mathrm{y}_{\mathrm{s}}$ for each fire streak. ${ }^{4}$ The final ingredient of the model is that each fire streak

\footnotetext{
${ }^{4}$ For the actual non-trivial distribution of $E_{\mathrm{s}}^{*}$ and $\mathrm{y}_{\mathrm{s}}$ in the transverse space as a function of collision centrality, and for the detailed discussion of normalisation issues in the comparison of the model to the experimental data discussed later in the text, the reader is referred to Ref. [31].
} 

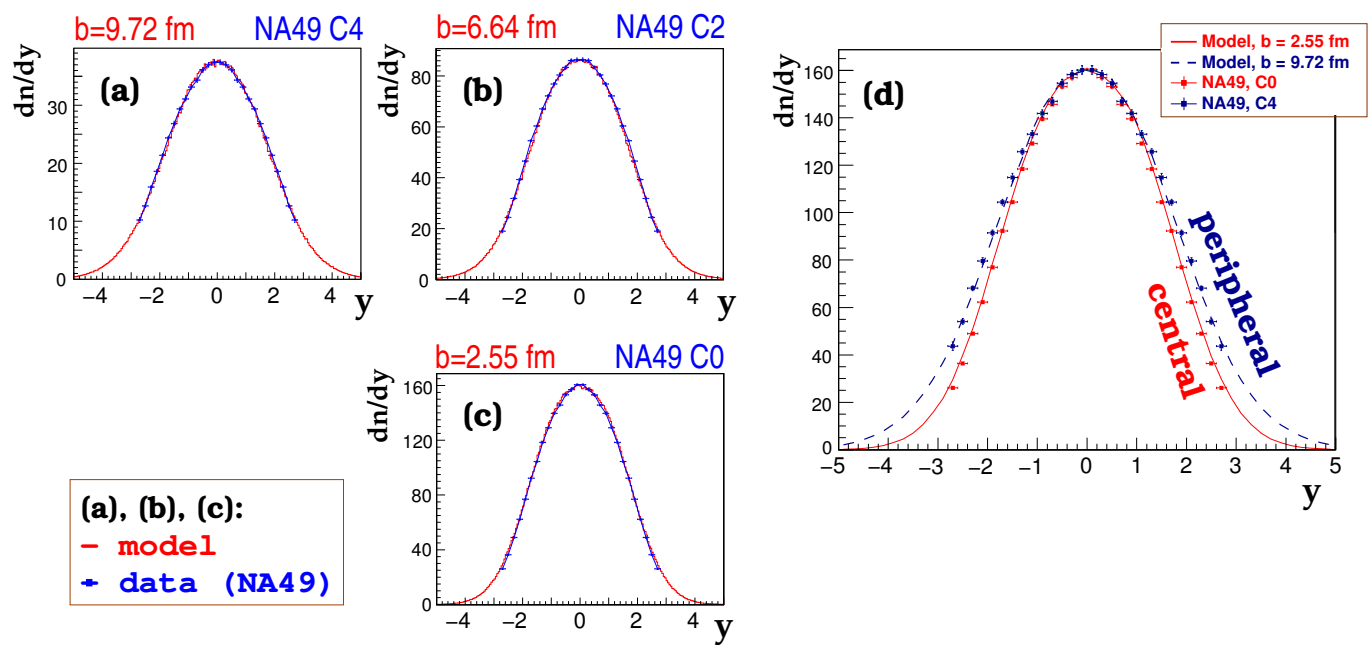

Figure 9. (a)-(c) Rapidity distributions of negatively charged pions measured by the NA49 experiment in three centrality classes, from the most peripheral (C4) to the most central $(\mathrm{C} 0) \mathrm{Pb}+\mathrm{Pb}$ collisions at $\sqrt{s_{N N}}=17.3 \mathrm{GeV}$, compared to the fire streak model [31]; experimental data are taken from Ref. [34]. (d) Shape comparison of rapidity spectra for two extreme centrality classes together with the fire streak model. The spectrum for peripheral collisions is renormalized at its peak to central collisions.

fragments independently into $n$ pions, according to the function [31]:

$$
\frac{\mathrm{d} n}{\mathrm{dy}} \sim A \cdot\left(E_{\mathrm{s}}^{*}-m_{\mathrm{s}}\right) \cdot \exp \left(-\frac{\left[\left(\mathrm{y}-\mathrm{y}_{\mathrm{s}}\right)^{2}+\epsilon^{2}\right]^{\frac{r}{2}}}{r \sigma_{\mathrm{y}}^{r}}\right)
$$

of pion rapidity y with free parameters $A, r, \sigma_{\mathrm{y}}$ independent of the fire streak and collision centrality, $\epsilon=0.01$, and other parameters describing properties of the fire streak stemming directly from the conservation rule. From the distribution of fire streak velocities, depicted as thick arrows in Fig. 8(d), and formula (5) one finds that on average, pions produced closer to the spectator system are moving faster.

Summing formula (5) over all fire streaks, one obtains the full rapidity spectrum of pions. The fit of the model to data from NA49 [34] for different centrality classes is shown in Fig. 9. It is apparent that the model describes well the dependence on centrality of both the yields of pions and shapes of pion rapidity spectra. This means that the longitudinal evolution of the system at CERN SPS energies can be interpreted as a pure consequence of local energy-momentum conservation.

Finally, it should be noted that apart from three measurements leading to the results in Fig. 8(b), an analysis of EM effects for Ar+Sc collisions in the NA61/SHINE experiment is ongoing [25].

\section{Summary}

New results on observables relevant for the strong interactions programme of the NA61/SHINE experiment were presented. They include the first ever differential measurements of $\phi$ meson production in $\mathrm{p}+\mathrm{p}$ collisions at 40 and $80 \mathrm{GeV}$ beam momentum and the first double-differential measurements at $158 \mathrm{GeV}$. These results were compared to world data on $\mathrm{p}+\mathrm{p}$ collisions showing consistency and 
superior accuracy of the new measurements. It was also demonstrated that none of the considered models is able to describe all the properties of $\phi$ production in the measured reactions. Finally, the comparison of $\mathrm{p}+\mathrm{p}$ to $\mathrm{Pb}+\mathrm{Pb}$ collisions shows a non-trivial system size dependence of the longitudinal evolution of hidden strangeness production, contrasting with that of other mesons. The latter observation emphasizes the importance of the analysis of $\mathrm{Be}+\mathrm{Be}, \mathrm{Ar}+\mathrm{Sc}$ and $\mathrm{Xe}+\mathrm{La}$ data collected by NA61/SHINE.

Regarding the search for the QCD critical point, it was shown that there is some evidence from NA49 for intermittency in "Si" $+\mathrm{Si}$ collisions at $158 \mathrm{~A} \mathrm{GeV}$ and none detected by NA49 in "C" $+\mathrm{C}$, $\mathrm{Pb}+\mathrm{Pb}$, nor by $\mathrm{NA61/SHINE}$ in $\mathrm{Be}+\mathrm{Be}$ interactions at the same energy. The analysis in $\mathrm{Ar}+\mathrm{Sc}$ reactions is ongoing.

Spectator-induced electromagnetic effects in pion emission bring information on the space-time position of the pion formation zone, which appears to be much closer to the spectator system for faster pions than for slower ones. On that basis, it has been demonstrated that the longitudinal evolution of the system at CERN SPS energies can be interpreted as a pure consequence of local energymomentum conservation.

\section{Acknowledgements}

This work was supported by the National Science Centre, Poland (grant numbers: 2014/14/E/ST2/00018, 2015/18/M/ST2/00125) and the Foundation for Polish Science - MPD program, co-financed by the European Union within the European Regional Development Fund.

\section{References}

[1] N. Abgrall et al. (NA61/SHINE), JINST 9, P06005 (2014)

[2] S. Afanasiev et al. (NA49), Nucl. Instrum. Meth. A 430, 210 (1999)

[3] S.V. Afanasiev et al. (NA49), Phys. Rev. C 66, 054902 (2002)

[4] C. Alt et al. (NA49), Phys. Rev. C 77, 024903 (2008)

[5] K. Werner, F. Liu, T. Pierog, Phys. Rev. C 74, 044902 (2006)

[6] T. Pierog, K. Werner, Nucl. Phys. B (Proc. Suppl.) 196, 102 (2009)

[7] C. Baus, T. Pierog, R. Ulrich, CRMC (Cosmic Ray Monte Carlo package)

[8] T. Sjöstrand, S. Mrenna, P. Skands, J. High Energy Phys. 05, 026 (2006)

[9] S. Bass et al., Prog. Part. Nucl. Phys. 41, 255 (1998)

[10] M. Bleicher et al., J. Phys. G 25, 1859 (1999)

[11] S. Afanasiev et al. (NA49), Phys. Lett. B 491, 59 (2000)

[12] V. Blobel et al., Phys. Lett. B 59, 88 (1975)

[13] C. Daum et al. (ACCMOR), Nucl. Phys. B 186, 205 (1981)

[14] D. Drijard et al., Z. Phys. C 9, 293 (1981)

[15] M. Aguilar-Benitez et al. (LEBC-EHS), Z. Phys. C 50, 405 (1991)

[16] V. Vovchenko, V.V. Begun, M.I. Gorenstein (2016), arXiv: 1512.08025 [nucl-th]

[17] C. Alt et al. (NA49), Phys. Rev. C 78, 044907 (2008)

[18] A. Aduszkiewicz et al. (NA61/SHINE), Eur. Phys. J. C 77, 671 (2017)

[19] N. Abgrall et al. (NA61/SHINE), Eur. Phys. J. C 74, 2794 (2014)

[20] T. Anticic et al. (NA49), Phys. Rev. Lett. 93, 022302 (2004)

[21] S. Johnson, B. Jacak, A. Drees, Eur. Phys. J. C 18, 645 (2001) 
[22] F.K. Diakonos et al., PoS CPOD2006, 010 (2006)

[23] N.G. Antoniou et al., Phys. Rev. Lett. 97, 032002 (2006)

[24] T. Anticic et al. (NA49), Eur. Phys. J. C 75, 587 (2015)

[25] A. Aduszkiewicz et al. (NA61/SHINE), CERN-SPSC-2017-038; SPSC-SR-221 (2017)

[26] L. Adamczyk et al. (STAR), Phys. Rev. Lett. 112, 162301 (2014)

[27] A. Rybicki, A. Szczurek, M. Kłusek-Gawenda, Acta Phys. Polon. B46, 737 (2015)

[28] A. Rybicki, PoS EPS-HEP2009, 031 (2009)

[29] H. Schlagheck (WA98), Nucl. Phys A663, 725 (2000)

[30] A. Rybicki et al., Acta Phys. Polon. Supp. 9, 303 (2016)

[31] A. Szczurek, M. Kiełbowicz, A. Rybicki, Phys. Rev. C95, 024908 (2017)

[32] W.D. Myers, Nucl. Phys. A296, 177 (1978)

[33] A. Trzcińska et al., Phys. Rev. Lett. 87, 082501 (2011)

[34] T. Antitic et al. (NA49), Phys. Rev. C86, 054903 (2012) 